\title{
NEGATIVE KINETIC ENERGY BETWEEN PAST AND FUTURE STATE VECTORS*
}

\author{
Daniel Rohrlich, Yakir Aharonov, $†$ Sandu Popescu, Lev Vaidman \\ Raymond and Beverly Sackler Faculty of Exact Sciences \\ School of Physics and Astronomy, Tel-Aviv University \\ Ramat-Aviv, Tel-Aviv 69978 Israel
}

\begin{abstract}
An analysis of errors in measurement yields new insight into classically forbidden quantum processes. In addition to "physical" values, a realistic measurement can yield "unphysical" values; we show that in sequences of measurements, the "unphysical" values can form a consistent pattern. An experiment to isolate a particle in a classically forbidden region obtains a negative value for its kinetic energy. It is the weak value of kinetic energy between past and future state vectors.
\end{abstract}

1. Introduction. When the word "quantum" first entered the language of physics, it meant a restriction on possible values of energy; and it is still axiomatic that the only observable values of a physical quantity are the eigenvalues of a corresponding quantized operator. When we obtain values that are not eigenvalues, we interpret them as errors. Still, measurements are uncertain in practice, and can even yield classically forbidden, "unphysical" values. We have uncovered remarkable regularities in the way that "unphysical" values can appear in sequences of measurements, suggesting that these values may not be unphysical at all. In quantum theory, it seems, not only are physical quantities not restricted: they can take

\footnotetext{
* Talk presented at the Conference on Fundamental Problems in Quantum Theory, Baltimore, MD, June 1994

$\dagger$ also Department of Physics, University of South Carolina, Columbia, SC 29208
} 
values outside the classically allowed range. Here we discuss this new effect in the context of barrier penetration by quantum particles.

Barrier penetration, such as tunnelling into a potential wall, is a classically forbidden quantum process. Quantum particles can be found in regions where a classical particle could never go: it would have negative kinetic energy. But the eigenvalues of kinetic energy cannot be negative. How, then, can a quantum particle "tunnel"? The apparent paradox is resolved by noting that the wave function of a tunnelling particle only partly overlaps the forbidden region, while a particle found within the forbidden region may have taken enough energy from the measuring probe to offset any energy deficit. Nevertheless, actual measurements of kinetic energy can yield negative values. Here, we present a model experiment in which we measure the kinetic energy of a bound particle to any desired precision. We then attempt to localize the particle within the classically forbidden region. The attempt rarely succeeds, but whenever it does, we find that the kinetic energy measurements gave an "unphysical" negative result; moreover, these results cluster around the appropriate value, the difference between the total and the potential energy. This consistency, which seems to come from nowhere - a background of errors - suggests strongly that the notion of a quantum observable is richer than generally realized. Previous papers making this suggestion analyze a measurement of $\operatorname{spin}^{1}$ and a quantum time machine ${ }^{2}$ as well as negative kinetic energy. ${ }^{3-4}$

2. Negative kinetic energy. Our example may be summarized as follows: we prepare a large ensemble of particles bound in a potential well, in an eigenstate of energy, and measure the kinetic energy of each particle to a given precision. 
Then we measure the position of each particle and select only those cases where the particle is found within some region "far enough" from the well - with "far enough" depending on how precisely the kinetic energy was measured. In almost all such cases, we find that the measured kinetic energy values are negative and cluster around the particular negative value appropriate to particles in the classically forbidden region. Also, the spread of the clustering is the characteristic spread for kinetic energy measurements with this device.

We begin with a particle trapped in a potential well. The Hamiltonian is $H=p^{2} / 2 m+V(x)$, with $V(x)=-V_{0}$ for $|x|<a$ and $V(x)=0$ for $|x|>a$. We prepare an ensemble of particles in the ground state, with energy $E_{0}<0:\left|\Psi_{i n}\right\rangle=\left|E_{0}\right\rangle$. Following von Neumann, ${ }^{5}$ we model a measurement of kinetic energy with an interaction Hamiltonian $H_{i n t}=g(t) P \frac{p^{2}}{2 m}$, where $P$ is a canonical momentum conjugate to the position $Q$ of a pointer on the measuring device. The time-dependent coupling constant $g(t)$ is nonzero only for a short time interval, and normalized so that $\int g(t) d t=1$. When the time interval is very short, we call the measurement impulsive. For an impulsive measurement, $H_{\text {int }}$ dominates the Hamiltonians of the measured system and the measuring device. Then, since $\dot{Q}=\frac{i}{\hbar}\left[H_{\text {int }}, Q\right]$, we obtain for the operator $Q$

$$
Q_{f i n}-Q_{i n}=\frac{p^{2}}{2 m}
$$

In an ideal measurement the position of the pointer is precisely defined, and so we read a precise value of kinetic energy. But in practice, measurements involve uncertainty. To model a source of uncertainty, we take the initial state of the pointer to be

$$
\Phi_{i n}(Q)=\left(\epsilon^{2} \pi\right)^{-1 / 4} e^{-Q^{2} / 2 \epsilon^{2}}
$$


The uncertainty in the initial position of the pointer produces errors of order $\epsilon$; when $\epsilon \rightarrow 0$ we recover the ideal measurement. Thus, any measured value is possible, although large errors are exponentially suppressed. There is no mystery in such errors; they are expected, given the uncertainty associated with the measuring device. Measurements can even yield negative values. The negative values may be unphysical, but they are part of a distribution representing the measurement of a physical quantity. They should not be thrown out, since they give information about the distribution and contribute to the best estimate of the peak value. Since these errors originate in the measuring device, and not in the system under study, it seems that they cannot depend on any property of the system. However, closer analysis of these errors reveals a pattern which clearly reflects properties of the system under study. The pattern emerges only after selection of a particular final state of the system.

Initially, the particle and device are in a product state $\Psi_{i n}(x) \Phi_{i n}(Q)$; after the interaction is complete, the state is $e^{-\frac{i}{\hbar} P p^{2} / 2 m} \Psi_{i n}(x) \Phi_{i n}(Q)$, in which the particle and the device are correlated. Now we consider kinetic energy measurements followed by a final measurement of position, with the particle found far outside the potential well. For the final state we choose a gaussian wave packet with its center far from the potential well,

$$
\Psi_{f i n}(x)=\left(\delta^{2} \pi\right)^{-1 / 4} e^{-\left(x-x_{0}\right)^{2} / 2 \delta^{2}}
$$

and we require $\delta>\alpha \hbar^{2} / m \epsilon$. The condition for the particle to be "far enough" from the potential well is

$$
\alpha x_{0}>>\left(\alpha^{2} \hbar^{2} / 2 m \epsilon\right)^{2}
$$


Since $\alpha^{2} \hbar^{2} / 2 m=\left|E_{0}\right|$, the expression in parentheses is the ratio of the magnitude of the effect, $\left|E_{0}\right|$, to the precision of the measurement, $\epsilon$. For more precise measurements of kinetic energy $(\epsilon \rightarrow 0)$, the final state is selected at increasing distances from the potential well $\left(x_{0} \rightarrow \infty\right)$.

The state of the measuring device after the measurement, and after the particle is found in the state $\Psi_{f i n}(x)$, is obtained by projecting the correlated state of the particle and measuring device onto the final state of the particle $\Psi_{f i n}(x)$. Apart from normalization, it is $\Phi_{f i n}(Q)=\left\langle\Psi_{f i n}\left|e^{-\frac{i}{\hbar} P p^{2} / 2 m}\right| \Psi_{i n}\right\rangle \Phi_{i n}(Q)$. For simplicity, we take $V(x)$ to be a delta-function potential $(a \rightarrow 0)$. Then $\Psi_{i n}(x)$ is $\sqrt{\alpha} \exp (-\alpha|x|)$. As an integral over $x$, the final state is

$$
\Phi_{f i n}(Q)=\int_{-\infty}^{\infty} d x e^{-\left(x-x_{0}\right)^{2} / 2 \delta^{2}} e^{-\frac{i}{\hbar} P p^{2} / 2 m} e^{-\alpha|x|} \Phi_{i n}(Q)
$$

up to normalization. Note that the exponential of $-i P p^{2} / 2 m \hbar$ acts to translate $Q$ in $\Phi_{i n}(Q)$. If we could ignore the part of the integral near $x=0$, we could replace $p^{2}$ with $-\alpha^{2}$ in Eq. (5), and the final state of the measuring device would be $\Phi_{f i n}(Q)=\Phi_{i n}\left(Q+\alpha^{2} \hbar^{2} / 2 m\right)$. We cannot ignore this part of the integral, but by choosing $x_{0}$ in $\Psi_{f i n}(x)$ to be large, we can suppress it. If we express $\Psi_{i n}(x)$ via its Fourier transform and replace the operator $p$ with its eigenvalue, we obtain (up to a normalizing factor)

$$
\Phi_{f i n}(Q)=\frac{\pi}{\hbar \alpha} e^{\alpha x_{0}-\alpha^{2} \delta^{2} / 2} \int d p \frac{e^{-p^{2} \delta^{2} / 2 \hbar^{2}-i p x_{0} / \hbar}}{\alpha^{2} \hbar^{2}+p^{2}} \Phi_{i n}\left(Q-p^{2} / 2 m\right)
$$

This integral has poles at $p= \pm i \alpha \hbar$; we evaluate it by integration on a contour including a line of $p$ with imaginary part $-i p_{0}$, for any $p_{0}>\hbar \alpha$. The integral in Eq. (6) then reduces to two terms: a pole term

$$
\Phi_{i n}\left(Q+\alpha^{2} \hbar^{2} / 2 m\right)
$$


and a correction term, the integral in Eq. (6) with $p$ replaced by $p-i p_{0}$. The pole term represents the measuring device with its pointer shifted to the negative value $-\alpha^{2} \hbar^{2} / 2 m$. A short computation (see Ref. 4) shows that the correction term can be made arbitrarily small by taking $x_{0}$ large, as in Eq. (4). For $x_{0}$ large, the final state of the measuring device shows the "unphysical" result $-\alpha^{2} \hbar^{2} / 2 m$ for the kinetic energy, up to a scatter $\epsilon$ characteristic of the device.

We thus obtain a correlation between position measurements and prior kinetic energy measurements: nearly all particles found far outside the potential well yielded negative values of kinetic energy. On the other hand, we could consider all particles that produced negative values of kinetic energy, and ask about their final position. We would find nearly all these particles inside the well. The correlation works one way only. Prior kinetic energy measurements on particles found far from the well cluster around a negative value, but position measurements on particles yielding negative values of kinetic energy cluster around zero. How do we interpret this one-way correlation?

3. Interpretation. Our example suggests that particles in a classically forbidden region have negative kinetic energy. The conventional interpretation of quantum mechanics has no place for negative kinetic energy. However, the conventional interpretation involves an assumption about how measurements are made. The conventional interpretation considers measurements on ensembles of systems prepared in an initial state, without any conditions on the final state of the systems. Such an ensemble, defined by initial conditions only, may be termed a pre-selected ensemble. By contrast, we consider measurements made on pre- and post-selected 
ensembles, defined by both initial and final conditions. The experiment of the previous section is an example of a measurement on a pre- and post-selected ensemble. It is natural to introduce pre- and post-selected ensembles in quantum theory: in the quantum world, unlike the classical world, complete specification of the initial state does not determine the final state.

Also, the measurements we consider are not ideal. Real measurements are subject to error. At the same time, the disturbance they make is bounded. These two aspects of non-ideal measurements go together. Suppose our measuring device interacts very weakly with the systems in the ensemble. We pay a price in precision. On the other hand, the measurements hardly disturb the ensemble, and therefore they characterize the ensemble during the whole intermediate time. Even non-commuting operators can be measured at the same time if the measurements are imprecise. When such measurements are made on pre- and post-selected ensembles, they yield surprising results. An operator yields weak values that need not be eigenvalues, or even classically allowed. ${ }^{1,6}$ The negative kinetic energy of the previous section is an example of a weak value. Another is a measurable value of 100 for a spin component of a spin-1/2 particle. $^{1}$

Let us briefly review how weak values arise. The initial wave function of the measuring device is $\Phi_{i n}(Q)$. After an impulsive measurement of an operator $C$ on an initial state $|a\rangle$ and projection onto a final state $|b\rangle$, the final state of the measuring device is $\left\langle b\left|e^{-i P C / \hbar}\right| a\right\rangle \Phi_{i n}(Q)=\sum_{i}\left\langle b \mid c_{i}\right\rangle\left\langle c_{i} \mid a\right\rangle \Phi_{i n}\left(Q-c_{i}\right)$. If $\Phi_{i n}(Q)$ is sharply peaked, then the various terms $\Phi_{i n}\left(Q-c_{i}\right)$ will be practically orthogonal. But suppose $\Phi(Q)$ has a width $\epsilon$. Its Fourier transform has a width in $P$ of $\hbar / \epsilon$. Small $|P|$ 
corresponds to a measuring device that is coupled weakly to the measured system. If $\epsilon$ is large, then $|P|$ is small, and we can expand the exponential $e^{-i P C / \hbar}$ to first order in $P$ to obtain $\left\langle b\left|e^{-i P C / \hbar}\right| a\right\rangle \Phi(Q) \approx\langle b|1-i P C / \hbar| a\rangle \Phi(Q) \approx\langle b \mid a\rangle e^{-i P C_{w} / \hbar} \Phi(Q)$. Here $C_{w} \equiv\langle a|C| b\rangle /\langle a \mid b\rangle$ is the weak value of the operator $C$ for the pre- and postselected ensemble defined by $\langle b|$ and $|a\rangle$.

The definition of a weak value provides us with a new and intuitive language for describing quantum processes. In our example, the operators of total energy $E$, kinetic energy $K$, and potential energy $V$ do not commute. Therefore, the classical formula $E=K+V$ applies only to their expectation values; and the expectation value of $K$ in any state is positive. However, the formula applies to weak values: $E_{w}=K_{w}+V_{w}$, and the weak value of $K$ is not necessarily positive. We know $E_{w}=E_{0}=-\alpha^{2} \hbar^{2} / 2 m$, since the pre-selected state is an energy eigenstate, and $V_{w}$ vanishes since the post-selected state is far from the potential well. Then $K_{w}=-\alpha^{2} \hbar^{2} / 2 m$, the "unphysical" obtained above in our example!

In our example, instead of the condition on the initial state of the measuring device $\left(\epsilon\right.$ large), we had a condition on the final state of the particle $\left(x_{0}\right.$ large and $\left.\delta>\alpha \hbar^{2} / m \epsilon\right)$. The price is that we must wait for increasingly rare events. As measurements of kinetic energy become more precise $(\epsilon \rightarrow 0)$, they disturb the particle more. To get negative kinetic energies, we must post-select particles further from the potential well $\left(x_{0} \rightarrow \infty\right)$. As the precision of the measurement increases, negative kinetic energies become less and less frequent; in the limit of ideal measurements, the probability vanishes, and so ideal measurements of kinetic energy never yield negative values. 
4. Conclusions. From the point of view of standard quantum theory, all that we have produced is a game of errors of measurement. Ideal measurements of kinetic energy can yield only positive values, since all eigenvalues of the kinetic energy operator are positive. But in practice, measurements are not exact, and even if their precision is very good, sometimes - rarely - they yield negative values. We have seen that if particles are subsequently found far from the potential well, the measured kinetic energy of these particles comes out negative. Consistently, large measurement "errors" did occur, producing a distribution peaked at the "unphysical" negative value $E_{0}$.

What special properties of non-ideal measurements led to this result? First, these measurements involve only bounded disturbances of particle position. Second, since their precision is limited, they can supply, "by error", the necessary negative values. These two properties are intimately connected: any measurement of kinetic energy causing only bounded changes of position must occasionally yield negative values for the kinetic energy. The change of $x$ due to the measurement is $\dot{x}=\frac{i}{\hbar} g(t)\left[x, P p^{2} / 2 m\right]$. $P$ and $p$ are unchanged during the measurement, so $x_{f i n}-x_{i n}=P p / m$. From here it follows that the change of $x$ is bounded only if the pointer is in an initial state with $P$ bounded, i.e. if the Fourier transform of $\Phi_{i n}(Q)$ has compact support. But then the support of $\Phi_{i n}(Q)$ is unbounded, ${ }^{7}$ which immediately implies a nonzero probability for the pointer to indicate negative values $(Q<0)$. Indeed, the "game of errors" displays a remarkable consistency, and this consistency allows negative kinetic energies to enter physics in a natural way. The concept of a weak value of a quantum operator gives precise meaning to the statement that the kinetic energy of a particle in a classically forbidden region is negative: namely, the weak value of the kinetic energy is negative. 
Acknowledgement. The research was supported by grant $425 / 91-1$ of the the Basic Research Foundation (administered by the Israel Academy of Sciences and Humanities) and by National Science Foundation grant PHY-8807812.

\section{References}

1. Aharonov, Y., D. AlBert \& L. VAIDMAN. 1988. Phys. Rev. Lett. 60: 1351 .

2. AHARONOV, Y., J. ANANDAN, S. POPESCU \& L. VAIDMAN. 1990. Phys. Rev. Lett. 64: 2965.

3. AHARONOV, Y., S. POPESCU, D. ROHRLICH, \& L. VAIDMAN. 1993. In Proc. 4th Int. Symp. Foundations of Quantum Mechanics, Tokyo, 1992. JJAP Series 9: 251.

4. AhARONOV, Y., S. POPESCU, D. ROHRLICH \& L. VAIDMAN. 1993. Phys. Rev. A48: 4084.

5. VON NEUMANN, J. 1983. Mathematical Foundations of Quantum Theory Princeton University Press. Princeton, New Jersey.

6. Aharonov, Y. \& L. VAIDMAN. 1990. Phys. Rev. A41: 11. AHARONOV, Y. \& D. ROHRLICH. 1990. In Quantum Coherence (Proceedings of the Conference on Fundamental Aspects of Quantum Theory, Columbia, South Carolina, 1989). J. S. Anandan, Ed. World-Scientific.

7. If the Fourier transform of $\Phi_{i n}(Q)$ has compact support, then $\Phi_{i n}(Q)$ is analytic. The two derivations of our result, via contour integration and via Taylor expansion of the exponential in Sec. 3, both require $\Phi_{i n}(Q)$ to be analytic. 